\title{
Manufacture of Epikeratophakia Lens
}

\author{
B. L. HALLIDAY \\ London
}

\begin{abstract}
Summary
Epikeratophakia lenses may be manufactured from donor cornea either with a lathe or using devices that make flat cuts on to previously deformed cornea. Details are given of a manufacturing technique using a cryolathe, including the storage and preparation of donor corneas and derivation of the formulae used to determine the radius of lathe cut.
\end{abstract}

Epikeratophakia has become established as a relatively safe surgical alternative for the correction of aphakia. Non surgical methods of correction with spectacles or contact lenses are generally preferable. If these methods are impractical and if intraocular lens implantation is contraindicated, then epikeratophakia may be appropriate. The technique has also been used in myopia and in keratoconus.

The surgery is relatively easy. Although techniques are still evolving, all involve the removal of epithelium from host cornea and cutting a peripheral groove to receive the epikeratophakia lens. The skills involved in conventional corneal grafting can be easily transferred to epikeratophakia and the postoperative management is similar to that of lamellar grafts.

In contrast, manufacture of lenses from donor cornea appears to be relatively difficult. Special equipment is needed to make the lenses, and formulae are required to calculate the effective refractive power of a manufactured lens. Some of the techniques of manufacture are discussed, lathing formulae are derived, and a detailed description of cryo lathing given.

\section{Techniques of Manufacture}

These fall into two broad categories. The donor cornea may either have a curved cut made on it, as with a lathe, (Fig. 1) or it may be cut flat across its surface, as with a guillotine (Fig. 2). In the latter case it is necessary to deform the cornea before cutting so that the flat cut results in the desired curved surface.

The advantages of lathing are that the required curvature is cut directly and therefore predictably, and that the high speed of rotation guarantees symmetry in the finished product. Existing small lathes, such as those used in contact lens manufacture may be modified quite easily to make them suitable. It is necessary to freeze the cornea to allow it to be cut with a steel or diamond tipped tool.

Freezing causes fractures in Bowmans membrane and probably kills all the keratocytes. ${ }^{1}$ Prior treatment of the cornea with a cryopreservative such as dimethylsulphoxide and using a controlled rate of freezing may allow keratocyte survival. ${ }^{2}$

The flat cut techniques are performed on cornea fresh from the eye bank so eliminate freezing damage. The keratocytes remain viable and this may result in quicker recovery of vision postoperatively. The presence of viable keratocytes may however make the epikeratophakia lens liable to rejection, which never occurs with cryolathed lenses. The flat cut may be made either with a mic-

Correspondence to: B. L. Halliday FRCS, Moorfields Eye Hospital, City Road, London, EC1V 2PD.

Presented at the Annual Congress of the Ophthalmological Society of the United Kingdom, April 1988. 
rokeratome using a high speed oscillating steel blade or may be made with an excimer laser. Commercial lens manfuacturing equipment using a microkeratome is available (BKS-1000, Allergan Medical Optics), but so far excimer laser manufacture is experimental.

\section{Calculation of Lathed Radius}

Interactions between the manufactured lens and host cornea will modify the final effective optical power. These unpredictable interactions, including relative dehydration by host endothelial cells and repopulation of the lens by host keratocytes, limit the value of purely theoretical calculations of lens power. Such calculations do however provide a base from which modifications can be made in the light of clinical experience.

There is a fundamental difference between lathing a contact lens and lathing an epikeratophakia lens. With a contact lens the back surface is lathed to fit on to the patient's cornea and the front surface radius is chosen to give the required refractive power. In contrast an epikeratophakia lens is cut only from behind; anteriorly Bowmans layer remains intact. This single cut therefore has to take account both of host keratometry readings and of the refractive power required. Figure 3 shows how the lathed lens changes shape when applied to host cornea. It is possible to calculate this change of shape and so calculate a theoretical power for a given epikeratophakia lens. Similar calculations


Fig. 1. Donor cornea (left) is supported on the lathe base (right) then the posterior lamellae are lathed away leaving a lens of the desired power. have been described for keratomileusis and, using different geometric principles, for epikeratophakia. ${ }^{3,4}$
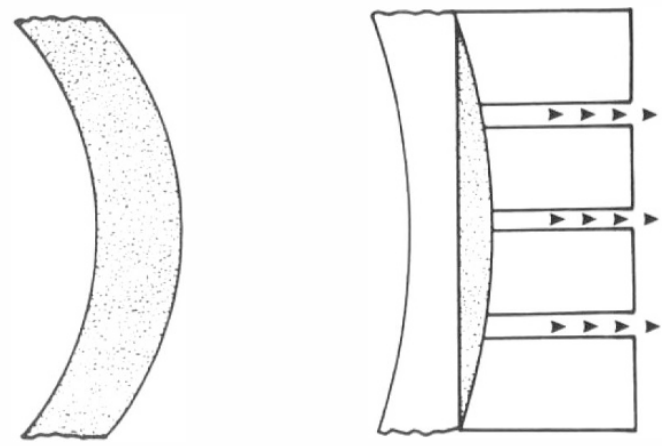

Fig. 2. Donor cornea (left) is sucked on to a die (right) to deform it. A flat tangential cut is made. On releasing suction a lens of the desired power remains.
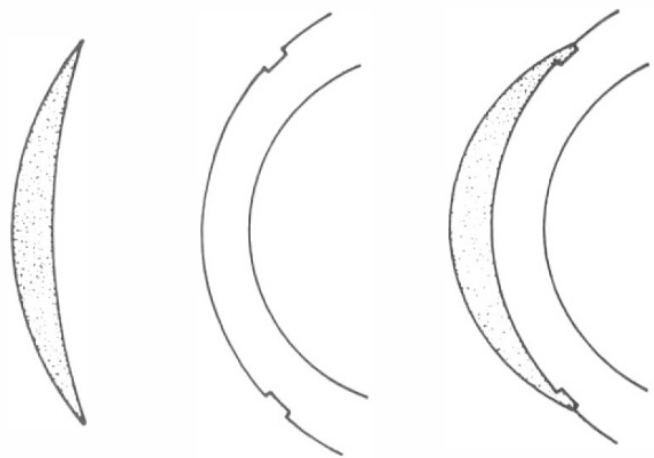

Fig. 3. An epikeratophakia lens (left) is sewn on to a cornea (centre). This deforms the lens (right) by a predictable amount.



Fig. 4. The geometry of an epikeratophakia lens may be expressed by the radii of curvature of the two surfaces $r 1$ and $r 2$ and by the overall diameter $2 A D$. 
Figure 4 shows a cornea that has been lathed. The front surface of the cornea still has its original radius, $\mathrm{r} 1$, of about $8 \mathrm{~mm}$. The back surface has been cut with a lathe set to a radius of $r 2$. The lathing has left the overall diameter of the lens equal to $2 \mathrm{AD}$.

From the diagram

$\mathrm{AB}^{2}+\mathrm{AD}^{2}=\mathrm{r}^{2}$

Therefore

$\mathrm{AB}=\sqrt{ }\left(\mathrm{rl}^{2}-\mathrm{AD}^{2}\right)$

From the diagram;

$(\mathrm{AB}+\mathrm{BC})^{2}+\mathrm{AD}^{2}=\mathrm{r} 2^{2}$

Therefore



Substituting into (2) for AB (from (1)) gives;

$\mathrm{BC}=\sqrt{ }\left(\mathrm{r} 2^{2}-\mathrm{AD}^{2}\right)-\sqrt{ }\left(\mathrm{rl}^{2}-\mathrm{AD}^{2}\right)$

Now from the diagram:

$\mathrm{r} 2+\mathrm{EF}=\mathrm{r} 1+\mathrm{BC}$

Therefore;

$\mathrm{EF}=\mathrm{r} 1-\mathrm{r} 2+\mathrm{BC}$

Substituting in (4) for BC (from (3)) gives

$\mathrm{EF}=\mathrm{r} 1-\mathrm{r} 2+\sqrt{ }\left(\mathrm{r} 2^{2}-\mathrm{AD}^{2}\right)-\sqrt{ }\left(\mathrm{r} 1^{2}-\right.$ $\mathrm{AD}^{2}$ )

It is therefore possible to calculate the lens thickness EF having fixed the values for the lens radius $\mathrm{AD}$, and for the radius of cut $\mathrm{r} 2$.

It is necessary to calculate how this lens changes shape when it is used clinically. As the lens is sewn in place its back radius becomes equal to that of host keratometry (Fig. 3). Calculation of the resulting front radius will give the effective power of the lens. For this calculation of change in lens shape it may be assumed that both the central thickness and the volume of the lens remain constant. On the diagrams constant volume is equivalent to constant cross sectional area and so it is with this that the calculation proceeds.

From the diagram the area of the lens may be given in terms of the sectors EBD and FCD and the triangle DBC:

Area $=\mathrm{EBD}+\mathrm{DBC}-\mathrm{FCD}$

Now these individual areas may be calculated:

$\mathrm{EBD}=1 / 2 \mathrm{rl}^{2}(\mathrm{ABD})$ where $\mathrm{ABD}$ is in radians $\mathrm{DBC}=1 / 2 \mathrm{AD}(\mathrm{BC})$

$\mathrm{FCD}=1 / 2 \mathrm{r} 2^{2}(\hat{A C D})$ where $A \hat{C D}$ is in radians
Therefore the area of the lens is given in:

Area $=1 / 2 \mathrm{rl}^{2}(\mathrm{ABD})+1 / 2 \mathrm{AD}(\mathrm{BC})-1 / 2 \mathrm{r} 2^{2}$

(AĈD)

Now the above angles can be expressed: '

$\mathrm{ABD}=\sin ^{-1}(\mathrm{AD} / \mathrm{r} 1)$

$\mathrm{ACD}=\sin ^{-1}(\mathrm{AD} / \mathrm{r} 2)$

Substituting into (7) from (8) and (9) gives

Area $=1 / 2 \mathrm{r} 1^{2}\left(\sin ^{-1}(\mathrm{AD} / \mathrm{r} 1)\right)+1 / 2 \mathrm{AD}(\mathrm{BC})-$ $1 / 2 \mathrm{r}^{2}\left(\sin ^{-1}(\mathrm{AD} / \mathrm{r} 2)\right)$

$\mathrm{BC}$ is known (3) and when this is substituted into (10), the area may be calculated knowing only the overall diameter of the lens (2AD), the base radius of the lathe ( $\mathrm{r} 1)$ and the radius of lathe cut $(\mathrm{r} 2)$.

The radius of lathe cut required for a given theoretical power of epikeratophakia lens follows from these calculations. An iterative computer program has been developed. Working to a given overall diameter the computer first makes a guess at a radius of lathe cut. Formulae (5) and (10) are then used to calculate the central thickness and cross sectional area of the resulting. lens.

The same formulae are then applied to work out the new shape of this lathed lens when applied to host cornea. Again an iterative technique is used. The central thickness of the lens is unchanged and the new back radius of the lens equals host keratometry, but of course the overall diameter of the lens reduces when it is placed on the host cornea (Fig. 3). The computer therefore guesses the new front radius and knowing central thickness can calculate the new overall diameter. It can then calculate the cross sectional area. Repeated guesses at front radius are made until the calculated cross sectional area equals that of the original lens. When it does the computer has effectively found the actual shape of lens on the host and can work out its optical power. This power is compared with the desired power and successive guesses made at the original radius of lathe cut until the required optical power is reached.

The above calculation gives a theoretical required radius of cut. Clinical experience has shown that the actual radius of cut needs to be flatter than this. An explanation may be that the donor lens is relatively thick and is further thickened by freezing. When the lens stabilises on the host it thins and so the power 
of the lens effectively reduces. The computer program has been modified with an evolving 'fudge factor' which is a simple percentage adjustment performed on the linear dimensions of the lathed lens before considering the change in shape as the lens is put on the eye.

\section{Design of Lens}

Once a radius of back cut has been decided lathing may be performed. The lens may be cut with this radius all the way to the edge, or more usually a wing may be made to facilitate suturing (Fig. 5). The wing is lathed to the same radius of curvature as the front surface of the lens; usually $8 \mathrm{~mm}$. Adding a wing increases the central thickness but this does not have a significant effect on the optical power.

Typical dimensions of the lathed lens are a wing thickness of $0.2 \mathrm{~mm}$, central thickness of between 0.5 and $0.75 \mathrm{~mm}$ and an overall diameter betwen 6 and $10 \mathrm{~mm}$. Central thickness depends on the power of the lens and the overall diameter. Lathed radii of curvature vary between $8 \mathrm{~mm}$ for the wings and for plano lenses (for keratoconus) and over $20 \mathrm{~mm}$ for some aphakic powers.

\section{Practical Manufacture}

The author uses a modified contact lens lathe to manufacture epikeratophakia lenses. The lathe is kept in a laminar flow cabinet to reduce the chance of airbourne contamination. A cylinder of carbon dioxide gas feeds cryo elements in the cutting tool and in the headstock of the lathe. An aluminium base cut to a radius of $8 \mathrm{~mm}$ supports the donor cornea which is held in place initially by surface tension and then by the adhesion of ice when the cornea is frozen. A diamond tipped cutting tool is used mounted on a double radius base of a design that allows two radii of cut, for the wing and for the optic zone, to be preset. A second cutting tool, mounted perpendicular on the lathe, may be used where needed to give a finishing diameter to the lens.

Donor corneas, obtained from our Eye Bank, are routinely stored in K-Sol (Coopervision-Cilco) at $4^{\circ} \mathrm{C}$ for between one and three weeks. Epithelium is removed with a

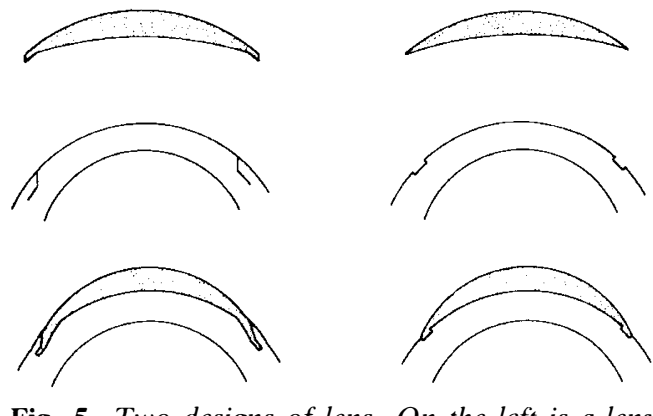

Fig. 5. Two designs of lens. On the left is a lens with a wing that tucks into a pocket in the cornea. On the right a lens without a wing sits on top of the cornea.

cotton tipped stick keeping the cornea moist to maintain uniform hydration. Partial drying could distort the cornea and so induce astigmatism in the finished lens. The cornea is then punched from behind with a trephine about $2 \mathrm{~mm}$ larger than the intended diameter of the lens. After soaking the cutting tool and the base of the lathe in a proprietary glutaraldehyde solution, the cornea is mounted and the lathe turned slowly allowing centration to be checked. After freezing to approximately $-40^{\circ}$ Celsius, lathing starts with initial cuts of about $0.1 \mathrm{~mm}$. For the final cut an advance of only $0.02 \mathrm{~mm}$ is made. The lens is then finished to the required diameter with the second cutting tool. The lens is replaced in $\mathrm{K}$ Sol and stored until use.

\section{Conclusions}

There are no insurmountable problems in setting up a cryolathe facility for the manufacture of epikeratophaia lenses. At current prices initial equipment costs would be covered by the savings over 100 commercially purchased lenses. After this time the cost of lens manufacture becomes extremely low. It is not known, nationally, how many patients would benefit from surgery; true level of demand may only become apparent when cheap lenses are available at every major referral centre.

\section{References}

${ }^{1}$ Binder PS, Zavala EY, Baumgartner SD, Nayak SK: Combined morphologic effects of cryolathing and lyophilization on epikeratoplasty lenticules. Arch Ophthalmol 1986, 104: 671-9 
${ }^{2}$ Lee TJ, Lce Wan W, Kash RL, Kratz KL, Schanzlin DJ: Keratocyte survival following a controlled rate freeze. Invest Ophthalmol Vis Sci 1985, 26: 1210-15.

${ }^{3}$ Barraquer JI: Calculations for refractive keratoplasty. Arch Soc Am Ophthal Optom 1970, 8: 103-64.

${ }^{4}$ Werblin PP and Klyce SD: Epikeratophalvia. The surgical correction of aphakia (i) Lasing of corneal tissue. Curr Eye Res 1981, 1: 123-9. 